\title{
Experiências do Desenvolvimento e da Validação de um Aplicativo Gamificado para Aprendizagem da Língua Brasileira de Sinais - LIBRAS
}

\author{
Luz H. B. Wessling ${ }^{1}$, Paulo Jr. Varela ${ }^{1}$, Adair J. Rohling ${ }^{1}$, Gustavo Y. Sato ${ }^{1}$ \\ ${ }^{1}$ Departamento de Informática - Universidade Tecnológica Federal do Paraná (UTFPR) \\ Caixa Postal 135 - 85.601-970 - Francisco Beltrão - PR - Brazil \\ luizw, paulovarela, adairrohling, gustavosato@utfpr.edu.br
}

\begin{abstract}
This paper presents an application to aid in the process of learning the Brazilian Sign Language - LIBRAS in a gamified way. The main idea is that both a deaf person without hearing loss can playfully learn to communicate in sign language by using application. The proposal software: (i) a application for mobile devices, therefore easily accessible; (ii) learn various contents, according to levels of difficulty; (iii) test the knowledge acquired and compare it with others users (gamification). The solution makes it possible to offer the inclusion and accessibility of people with hearing disabilities and their communication with people without disabilities.
\end{abstract}

Resumo. Este artigo apresenta uma aplicação para auxilio no processo de aprendizagem da Lingua Brasileira de Sinais - LIBRAS de forma gamificada. A ideia central é que tanto pessoa surda como pessoa sem deficiência auditiva possam aprender de forma lúdica a se comunicar em linguagem de sinais pelo uso do aplicativo. A ferramenta oferece: (i) uma aplicação para dispositivos móveis, portanto de fácil acesso; (ii) aprender diversos conteúdos, conforme niveis de dificuldade; (iii) testar os conhecimentos adquiridos e comparar com outros usuários (gamificação). A solução propicia ofertar a inclusão e a acessibilidade de pessoas com deficiência auditiva e a sua comunicação com pessoas sem deficiência.

\section{Introdução}

A aceleração do uso de tecnologias no meio educacional tem oportunizado o desenvolvimento de soluções para diversos tipos de público. As mais diversas formas de tecnologias propiciam a inclusão de alunos, e se torna essencial no processo de ensino-aprendizagem [Nascimento 2017]. Neste contexto, é possível observar na literatura algumas aplicações inclusivas, tais como: (i) deficiência auditiva [Silva 2002] [Schneider 2006] [Secco 2009] [Branco Neto 2009] [Rodrigues 2010]; (ii) deficiência visual [Nogueira 2011]; (iii) deficiência cognitiva [Franciscato 2007] [Sousa 2012] [Alves 2012] [Oliveira 2019]; e, (iv) deficiência física [Castro Júnior 2008]. Diante disso, sabe-se que a evolução do formato de ensino tradicional para um ensino mais inovador, que chame a atenção do aluno se faz essencial. Neste ponto, observam-se diversas lacunas de desenvolvimento de ambientes inovadores para o processo de ensino-aprendizagem em diversas áreas da inclusão social.

O desenvolvimento de novas ferramentas inclusivas pode vislumbrar novas perspectivas para o ensino de Libras, tendo como objetivo, a incorporação na prática diária e 
interativa da linguagem [Fiorio 2019]. Um outro ponto a ser levado em consideração, é que tanto um aluno ouvinte como um aluno com deficiência auditiva pode fazer dessas ferramentas, pois tratam de conteúdos análogos. E, por conseguinte o uso da gamificação torna a ferramenta em um ambiente prazeroso e lúdico no processo de aprendizagem dos conteúdos.

A gamificação é o uso de técnicas e design de jogos que utilizam de mecânicas e pensamentos orientados a jogos, que visam enriquecer diversos contextos e fazer com que os usuários utilizem jogos para aprender conteúdos de forma atrativa [Takahashi 2010]. $\mathrm{Na}$ área educacional a gamificação é uma forma de promover um processo de aprendizagem mais dinâmico, rápido e agradável. Além do mais, a literatura indica que pela gamificação os alunos conseguem: (i) absorver e reter os conteúdos; (ii) transmitir o conhecimento de uma forma mais clara; (iii) aumentar o diálogo e apresentação de narrativas; (iv) aumentar os graus de desafios e aprofundamento nos conteúdos; (v) menor taxa de reprovação [Mendonça 2016].

A contribuição principal deste artigo é disponibilizar uma ferramenta de software gamificada para auxilio na aprendizagem de Libras, tanto para alunos com deficiência auditiva como para alunos que não possuam deficiência. Em correlato, visa estimular que professores adotem a ferramenta como auxiliar para romper as barreiras convencionais da sala de aula tradicional, e fazer com que os alunos aumentem o aprendizado em Libras e consequentemente o seu rendimento escolar pelo uso do ambiente lúdico e gamificado.

Este artigo está estruturado da seguinte forma: Na seção 2 são apresentados os materiais e métodos utilizados, na seção 3 são evidenciados os resultados obtidos e na seção 4 é apresentado um comparativo com a literatura. E, por fim, na seção 5 as conclusões e as indicações de trabalhos futuros.

\section{Materiais e Métodos}

Inicialmente foi criado e aplicado um conjunto de questionamentos para professores e interpretes de Libras, bem como, para estudantes com deficiência auditiva, com o intuito de extrair os requisitos e identificar as principais necessidades e funcionalidades, ou seja, a definição do escopo da aplicação. Diante disso, foram desenvolvidos representações gráficas (diagramas) para um melhor entendimento da estrutura e funcionalidades. A modelagem escolhida foi a Unified Modelling Language - UML, que uma linguagem padrão para elaboração da estrutura de softwares [Guedes 2018]. No campo científico, este artigo utiliza procedimento de estudo de caso, com objetivo exploratório e finalidade aplicada.

Na Figura 1 é apresentado o diagrama de caso de uso, que representa as principais funcionalidades do aplicativo, que foram baseados nos seguintes requisitos funcionais: (i) proporcionar um cadastro de usuário para conseguir efetuar a rastreabilidade do desempenho; (ii) permitir a visualização dos rankings de desempenho do usuários; (iii) permitir que o usuário selecione os conteúdos que queira aprender; (iv) possibilitar que o usuário escolha os níveis de dificuldade dos conteúdos; e, (v) gerar testes de conhecimento sobre os assuntos do aprendizado. Além disso, a documentação completa do software pode ser encontrada em http://github.com/liquiz e o aplicativo pode ser baixado e usado gratuitamente na loja de aplicativos oficial da Google ${ }^{1}$.

\footnotetext{
${ }^{1}$ https://play.google.com/store/apps/details?id=com.liquiz.librasliquiz
} 


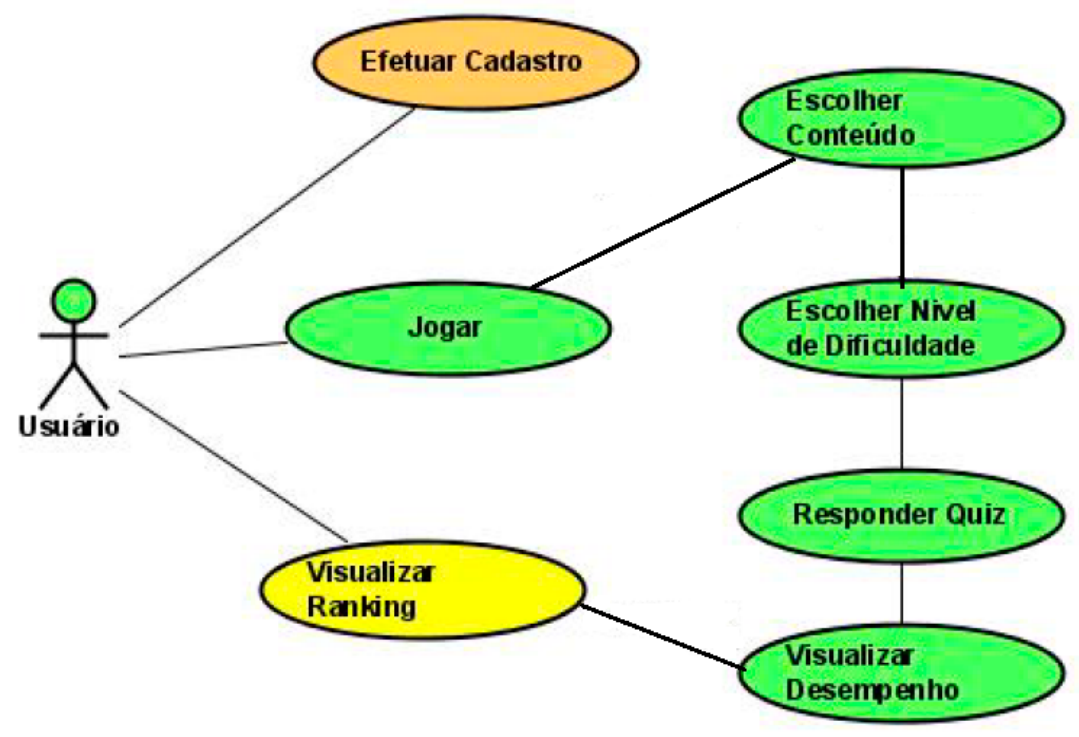

Figura 1. Diagrama de Caso de Uso

Para o desenvolvimento do aplicativo foram delineadas quatro fases que evidenciam a construção do software, como pode ser visto na Figura 2.

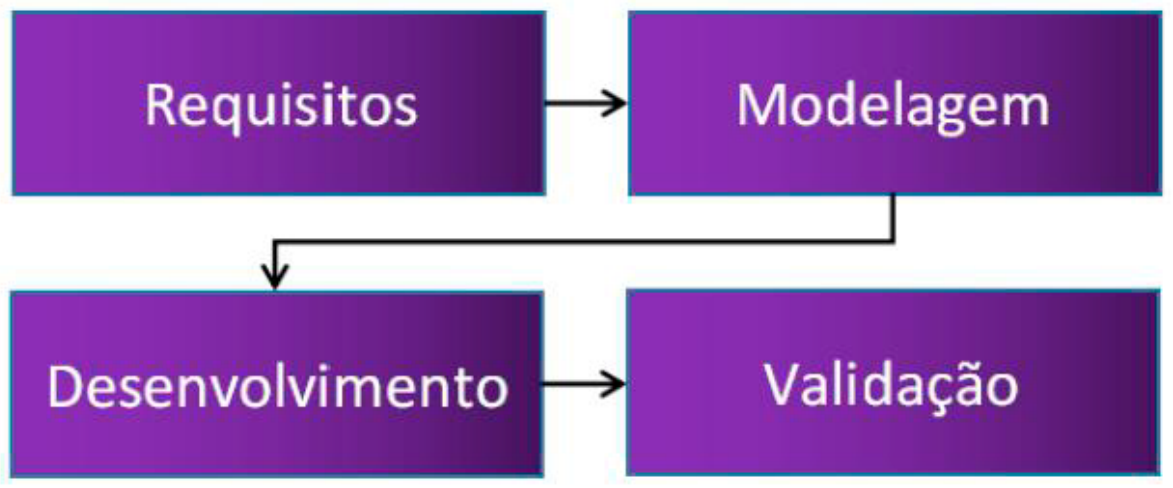

Figura 2. Fases de Desenvolvimento do LIQUIZ

Na primeira fase, são levantados e analisados os requisitos para chegar ao escopo do projeto. Neste caso, são organizadas as bases de imagens, vídeos, frases, perguntas e respostas em Libras e em Língua Portuguesa. Posteriormente, são desenvolvidos os diagramas da ferramenta na UML. Estes diagramas servem de base para o desenvolvimento do banco de dados e da programação. Neste ponto, também são desenvolvidas telas do aplicativo no formato de protótipo. A terceira fase, consiste do desenvolvimento do aplicativo, ou seja, onde é criado o banco de dados e efetuada a codificação em linguagem de programação, observando as regras do jogo e efetuando os testes iniciais. Por fim, a fase de validação, é onde ocorrem os testes do protótipo com usuários com deficiência auditiva e ouvintes para obtenção de feedback com o intuito de ajustar, corrigir e melhorar o aplicativo antes da disponibilização, o detalhamento dessas etapas serão apresentados na seção 3 .

No processo de desenvolvimento do aplicativo foi utilizado o Astah Professional 
na elaboração dos diagramas da UML, o Astah é um ambiente que possibilita a construção de todos os diagramas que são preconizados pelo Object Management Group - OMG [Guedes 2018]. Para o banco de dados utilizamos o MySql, que é um sistema gerenciador de banco de dados popular e gratuito. Quanto a linguagem de programação foi utilizado o Java no ambiente de programação Android Studio. O aplicativo é compatível com o sistema operacional Android que está presente em mais de $80 \%$ dos usuários de smartphones [Data 2019].

\section{Resultados}

O aplicativo possui funcionalidades organizadas em menus, são apresentadas as opções de cadastros, jogar e visualizar rankings. Na tela de cadastro, é possível realizar o cadastramento e alterações do usuário. Na opção de jogar, são exibidas as opções de conteúdo e nível de dificuldade. E na opção de visualizar ranking, o usuário pode verificar qual o seu nível de aprendizado em relação a outros usuários.

Para jogar o LIQUIZ, o usuário pode escolher o conteúdo do aprendizado por assunto, tais como: objetos, lugares, cumprimentos, animais, frutas, relacionamentos, pessoas, entre outros. Após a escolha do assunto é solicitado ao usuário qual o nível de dificuldade desejado, que são: iniciante, intermediário e avançado. Neste caso, os conteúdos do nível iniciante estão atrelados aos significados das palavras na tradução direta de Língua Portuguesa para Libras. No nível intermediário são estudadas a formação de frases, ou seja, o uso de um conjunto de sinais para formação mínima de uma frase. No nível avançado, são disponibilizados conteúdos através de vídeos que indicam uma comunicação mais avançada, tal como, a conversação entre dois indivíduos, que o usuário tem que interpretar.

Na Figura 3 é possível observar telas no nível iniciante, onde o usuário observa a imagem e escolhe entre a lista de opções a resposta correta (Figura 3 a). Caso o usuário escolha a resposta correta, o jogo vai informar o acerto (Figura 3 b), e caso a opção escolhida seja errada será informada que o usuário errou e qual seria a resposta correta (Figura 3 c).

Ao final de cada rodada do jogo (composto por 10 ou 15 questões aleatórias e conforme o assunto) é exibido o resultado atingido pelo usuário naquele teste. Neste caso, em correlato é mostrado ao usuário qual a posição do ranking que ele atingiu com o seus desempenho. Após a apresentação do relatórios de desempenho o usuário pode escolher continuar o jogo com outro assunto ou nível mais avançado ou refazer o quiz para melhorar o desempenho (neste caso, não serão os mesmos questionamentos, porque existe um banco de questões que são selecionadas de forma randômica a cada rodada).

O jogo faz uso de imagens, vídeos e textos que representas os sinais em Libras. Isso se faz necessário para deixar o jogo mais atrativo e interativo e com materiais multimídia houve uma maior instigação pelo grupo de validação.

Ao final, o aplicativo LIQUIZ foi colocado em validação em ambiente real. O aplicativo foi testado com diferentes tipos de público, tais como: alunos surdos e ouvintes de graduação, alunos surdos e ouvintes de séries iniciais (crianças de 6 a 9 anos), interpretes e professores de Libras. Neste contexto, as principais contribuições e percepções destacadas foram: 


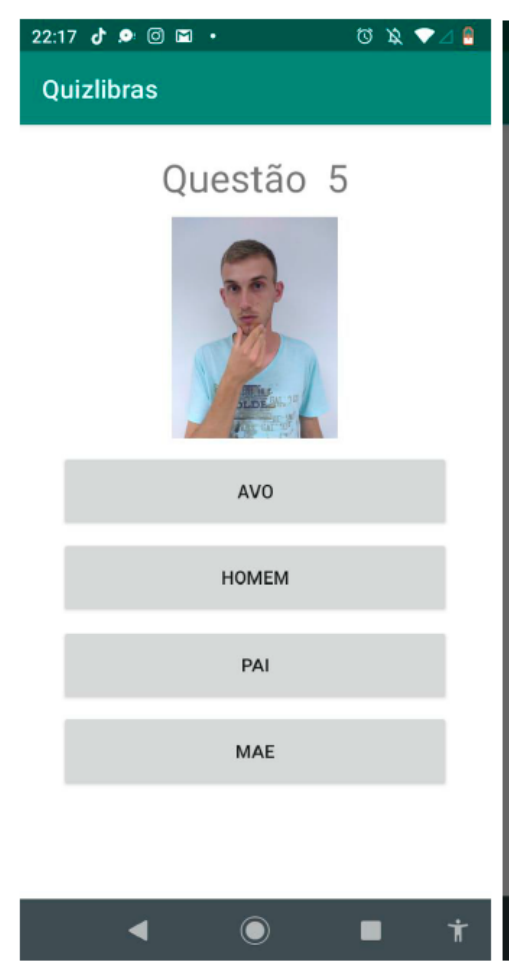

(a) Tela de Opções

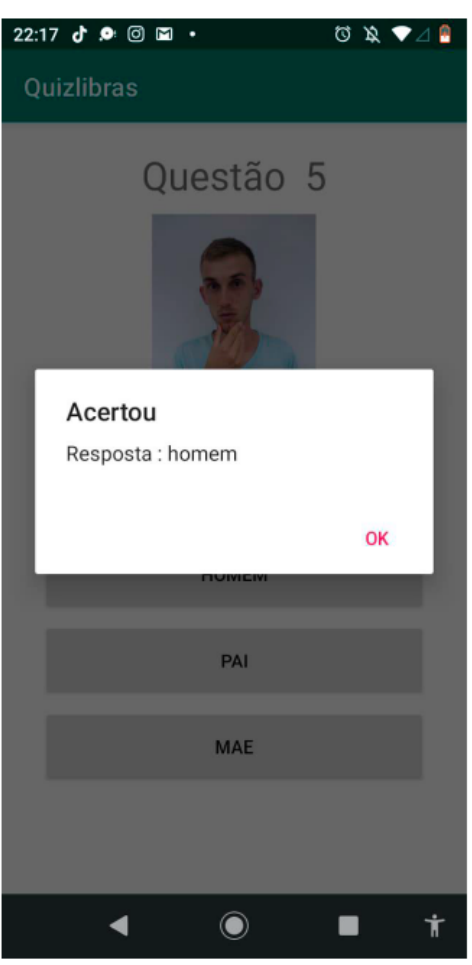

(b) Opção Correta

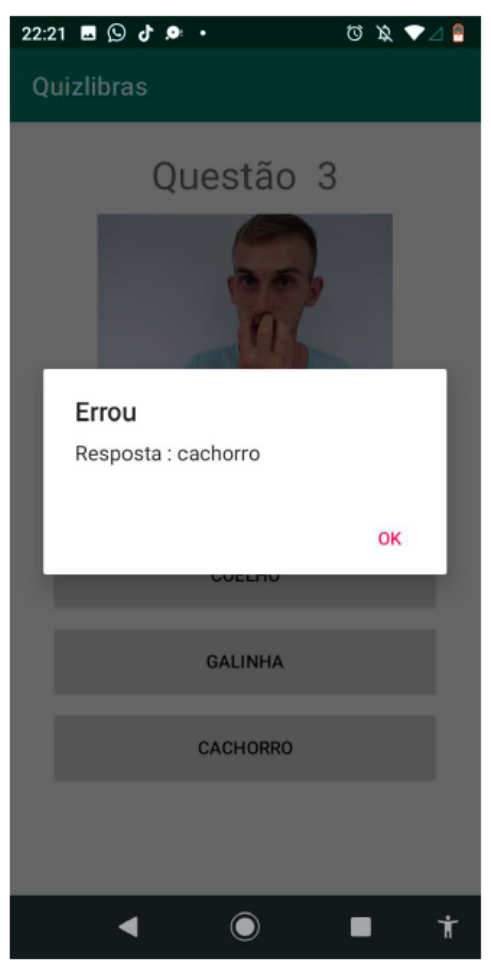

(c) Opção Errada

Figura 3. Telas do Aplicativo Liquiz

- (i) para alunos de graduação que possuem deficiência auditiva e já conhecem Libras, o jogo se mostrou mais uma ferramenta de apoio e de entretenimento, já que a grande maioria domina a língua de sinais;

- (ii) para alunos de graduação ouvintes a aplicação se mostrou interessante e atraente, para os indivíduos que querem se comunicar com colegas e amigos surdos;

- (iii) na aplicação com crianças surdas e ouvintes observamos os maiores efeitos, pois como ainda estão em fase alfabetização o jogo se mostrou atraente e produtivo, fazendo com que os alunos se interessassem mais pelo assunto;

- (iv) para os interpretes e professores de Libras a aplicação se mostrou eficiente, robusta e passível de ser aplicada no processo de aprendizagem de Libras como uma ferramenta auxiliar e lúdica, ou seja, sair do modelo tradicional de ensino, e oportunizar aos alunos as possibilidades de uso de tecnologias da informação e comunicação para compreender melhor os conteúdos desenvolvidos em sala de aula.

Além disso, para os professores o potencial da ferramenta está no fato de poder diversificar conteúdos, adaptação das aulas e acessibilidade do aluno. Um outro relato importante a ser destacado é que segundo os usuários que validaram o aplicativo, a usabilidade do software é simples e intuitiva, indo ao encontro aos relatos que preconizam que a interface humano-computador é de suma importância para a aceitabilidade de um software pelo público [Nielsen 2014]. Outro relevante destaque é avaliação altamente positiva (atualmente em 4.9 de no máximo 5) e os comentários realizados pelos usuários 
que usaram o aplicativo por meio da Play Store da Google. Diante disso, percebe-se que a aplicação LIQUIZ tem diversas possibilidades de utilização no processo de aprendizagem de Libras, principalmente com alunos de séries iniciais e ouvintes que queiram aprender e praticar a Língua Brasileira de Sinais.

\section{Comparação com a Literatura}

Com o intuito de verificar as características principais da nossa proposta, foi realizado um estudo comparativo na literatura buscando diferentes ferramentas e bibliotecas com foco na aprendizagem de LIBRAS e com objetivos diversos e suporte à várias linguagens. $\mathrm{O}$ resultado desse comparativo é demonstrado na Tabela 1.

Tabela 1. Comparativo com a Literatura

\begin{tabular}{|l|c|c|c|c|c|}
\hline \multicolumn{1}{|c|}{ Literatura } & Gamificação & Aplicativo & Educacional & Jogo & Multimídia \\
\hline [Silva 2002] & & & $\mathrm{X}$ & & $\mathrm{X}$ \\
\hline [Schneider 2006] & & & $\mathrm{X}$ & & $\mathrm{X}$ \\
\hline$[$ Branco Neto 2009] & & & $\mathrm{X}$ & & $\mathrm{X}$ \\
\hline$[$ Secco 2009] & & $\mathrm{X}$ & & $\mathrm{X}$ \\
\hline$[$ Rodrigues 2010] & & & $\mathrm{X}$ & $\mathrm{X}$ & $\mathrm{X}$ \\
\hline$[$ Santos 2014] & $\mathrm{X}$ & & $\mathrm{X}$ & $\mathrm{X}$ & $\mathrm{X}$ \\
\hline [Rocha 2019] & & $\mathrm{X}$ & $\mathrm{X}$ & $\mathrm{X}$ & $\mathrm{X}$ \\
\hline Nossa Proposta & $\mathbf{X}$ & $\mathbf{X}$ & $\mathbf{X}$ & $\mathbf{X}$ & $\mathbf{X}$ \\
\hline
\end{tabular}

Na Tabela 1 é possível observar algumas semelhanças e diferenças entre alguns trabalhos analisados na literatura sobre ferramentas computacionais em Libras. Neste caso, observa-se que os trabalhos desenvolvidos por [Silva 2002] [Schneider 2006] [Branco Neto 2009] [Secco 2009] são mais voltadas para a tradução de Libras de forma educacional, mas possuem atributos multimídia e foram desenvolvidos para ambientes desktop e web. Já a proposta de [Rodrigues 2010] apresenta um jogo educacional com material multimídia em ambiente web. Substancialmente o trabalho de [Santos 2014] apresenta um software com gamificação para o ensino de Libras, mas não disponibilizado no formato de aplicativo. E, [Rocha 2019] disponibiliza um jogo educacional em formato de aplicativo para ensino de química para pessoas com deficiência auditiva.

O diferencial principal da aplicação LIQUIZ, apresentada neste artigo está no fato de além de ser um aplicativo com fins educacionais em formato de jogo e com recursos multimídia, além de possuir características de gamificação, que faz com que nossa ferramenta se torne atrativa e usual.

\section{Conclusões}

A necessidade da inclusão e acessibilidade de alunos surdos é uma das áreas de pesquisa e desenvolvimento que existem diversas lacunas, onde a informatização quando aplicado de forma adequada pode ser um recurso essencial. Com isso, é de suma importância desenvolver ferramentas que auxiliem todos os processos do ensino, da aprendizagem e da aplicação da Língua Brasileira de Sinais. O aplicativo LIQUIZ permite a aprendizagem de Libras de forma gamificada, levando aos usuários uma forma lúdica de aprendizagem de conteúdos através de um jogo educacional.

Quanto a utilidade da ferramentas no ambiente educacional percebeu-se que a ferramenta se mostrou viável e aplicável para alunos e professores. Vale destacar que o 
aplicativo obteve uma alta taxa de aceitação entre alunos de séries iniciais e de alunos que não possuem deficiência auditiva, indicando que existem possibilidades de aplicação principalmente com estes públicos.

Fica notório que ferramentas computacionais que auxiliam o professor e aluno no ensino dos conteúdos da Língua Brasileira de Sinais são importantes. Neste caso, podemos citar como exemplo o uso da nossa aplicação como apoio para demonstrar aos alunos as diferentes perspectivas que um conteúdo pode ser trabalhado. Além do mais, incentivar a aprendizagem de Libras por uma ferramenta gamificada fomenta as discussões entre aluno-professor e aluno-aluno.

A ferramenta foi testada e validada em um grupo de usuários, e continua em aprimoramento para ser disponibilizada. Em uma próxima versão propõe-se trabalhar com uma arquitetura orientada à serviços. Pretende-se implementar novos níveis de dificuldade e possibilidade do professor gerenciar o conteúdo de uma turma em especifico, por exemplo. Além disso, realizar uma pesquisa com os usuários do sistema (professores e alunos) com maior amplitude para verificar os benefícios e limitações da ferramenta no processo de ensino-aprendizagem.

\section{Referências}

Alves, L.; Pereira-Guizzo, C. d. S. P. T. T. X. (2012). Jogos digitais na área da comunicação aumentativa e alternativa: delineando novos espaços interativos para crianças com paralisia cerebral. Revista da Associação de Tecnologia Educacional, $31: 37-52$.

Branco Neto, W. C.; Lorenzini, I. P. (2009). Sistema tutor inteligente para auxílio na alfabetização de crianças surdas em um contexto bilíngüe. XV Workshop de Informática na Escola, Bento Gonçalves, $R S$.

Castro Júnior, H. P.; Almeida, M. J. M. M. K. S. O. A. I. A. (2008). O software "brincando com leitura": estimulação de habilidades para a aprendizagem de leitura e escrita de crianças com deficiência. XIV Workshop de Informática na Escola, Belém, PA.

Data, I. B. (2019). Most popular mobile os 1999-2019. Gartner Reports.

Fiorio, R.; Varela, P. A. M. . S. J. (2019). Linguisticun: Uma ferramenta de auxílio ao ensino da língua portuguesa e à linguística computacional. Simpósio Brasileiro de Informática na Educação - SBIE.

Franciscato, T. F.; Canal, P. A. (2007). Argot - software para ensino de inglês para pessoas com síndrome de down. XVIII Simpósio Brasileiro de Informática na Educação, São Paulo, SP.

Guedes, G. T. A. (2018). UML 2 - Uma abordagem Prática. Novatec, $3^{\mathrm{a}}$ ed edition.

Mendonça, B. (2016). O que é a gamificação e como ela funciona? Disponível em: https://www.edools.com/o-que-e-gamificacao/ Acesso em: 10 de junho de 2021.

Nascimento, C. A. (2017). O uso do software gcompris como ferramenta pedagógica no processo de ensino e aprendizagem em uma perspectiva inclusiva. Dissertação (Mestrado em Educação) - Universidade de Brasília, Brasília, DF.

Nielsen, J.; Budiu, R. (2014). Usabilidade Móvel. Elsevier, $1^{\mathrm{a}}$ ed edition. 
Nogueira, A. S.; Nielsen, R. J. (2011). Homero software: Auxiliando o ensino de portadores de cegueira. XXII Simpósio Brasileiro de Informática na Educação, Aracajú, SE.

Oliveira, U. P.; Thereza, W. B. (2019). Avaliação de softwares educacionais: Uma abordagem mais inclusiva para alunos com transtorno do espectro autista. Workshop de Informática da Escola - WIE.

Rocha, K. N.; Almeida, N. M. S. C. R. G. S. L. F. M. S. (2019). Q-libras: um jogo educacional para estimular alunos surdos à aprendizagem de química. Revista Educação Especial, 32.

Rodrigues, Y. W. S.; Silva, N. G. R. S. E. V. S. D. S. D. F. A. J. G. M. O. G. A. S. A. C. (2010). Saeps: Uma ferramenta de apoio à alfabetização do surdo. XII Simpósio Brasileiro de Informática na Educação, João Pessoa, PB.

Santos, L. C. M. Miranda, T. I. M. A. S. C. S. M. M. C. F. P. P. C. R. (2014). Um jogo para aprender libras e português nas séries iniciais utilizando a tecnologia da realidade aumentada. Simpósio Brasileiro de Informática na Educação - SBIE.

Schneider, A. R. de A.; Nedel, L. (2006). Sistema de animação de humanos virtuais voltado para o ensino de libras. XVII Simpósio Brasileiro de Informática na educação, Brasília, DF.

Secco, R.L.; Silva, M. (2009). Proposta de um ambiente interativo para aprendizagem em libras gestual e escrita. XX Simpósio Brasileiro de Informática na Educação, Florianópolis, $S C$.

Silva, A. C. (2002). Karytu: Um software para o letramento de criança surda sob a ótica bilíngüe. XIII Simpósio Brasileiro de Informática na Educação, Porto Alegre, RS.

Sousa, F. R. M.; Costa, E. A. B. C. T. H. C. (2012). Worldtour: Software para suporte no ensino de crianças autistas. XXIII Simpósio Brasileiro de Informática na educação, Rio de Janeiro, RJ.

Takahashi, D. (2010). Gamification gets its own conference. Venture Beat. 